Check for updates

Cite this: Chem. Sci., 2018, 9, 2105

\title{
A DNA-conjugated small molecule catalyst enzyme mimic for site-selective ester hydrolysis $\dagger$
}

\author{
Moira L. Flanagan, A. Emilia Arguello, Drew E. Colman, Jiyeon Kim, Jesse N. Krejci, \\ Shimu Liu, Yueyu Yao, Yu Zhang and David J. Gorin (D) * \\ The challenge of site-selectivity must be overcome in many chemical research contexts, including selective \\ functionalization in complex natural products and labeling of one biomolecule in a living system. Synthetic \\ catalysts incorporating molecular recognition domains can mimic naturally-occurring enzymes to direct \\ a chemical reaction to a particular instance of a functional group. We propose that DNA-conjugated \\ small molecule catalysts (DCats), prepared by tethering a small molecule catalyst to a DNA aptamer, are \\ a promising class of reagents for site-selective transformations. Specifically, a DNA-imidazole conjugate \\ able to increase the rate of ester hydrolysis in a target ester by $>100$-fold compared with equimolar \\ untethered imidazole was developed. Other esters are unaffected. Furthermore, DCat-catalyzed \\ hydrolysis follows enzyme-like kinetics and a stimuli-responsive variant of the DCat enables \\ programmable "turn on" of the desired reaction.
}

Received 20th October 2017
Accepted 10th January 2018

DOI: $10.1039 / \mathrm{c} 7 \mathrm{sc} 04554 a$

rsc.li/chemical-science

intrinsic reactivity and direct a reaction to other sites. Many further developments in site-selective catalysis have drawn inspiration from nature. ${ }^{4}$ Protein enzymes recognize their substrates and catalyze reactions at a specific site; for example, proteases can hydrolyze one amide bond in the presence of many others, ${ }^{5}$ and restriction endonucleases sever the DNA backbone only at specific sequence locations. ${ }^{6}$ One factor contributing to enzyme selectivity is that binding to the target increases the effective concentration of the enzyme active site and the substrate, which in turn increases the rate of catalysis.

In analogy to biological enzymes, synthetic and semisynthetic catalysts incorporating molecular recognition elements to promote substrate binding and accelerate a desired reaction have been investigated. ${ }^{7}$ Site-selectivity within vancomycin was demonstrated using peptide catalysts incorporating a vancomycin-binding domain ${ }^{7 a}$ and site-selectivity for protein labeling on live cell surfaces has been achieved using a reagent modularly assembled from a reactive small molecule catalyst and an antibody binding domain. ${ }^{8}$ Like proteins, nucleic acids may fold into three-dimensional structures that confer a specific function, with the additional advantages that they may be evolved to bind nearly any target de novo, synthesized easily and cheaply, and denatured reversibly. ${ }^{9}$ Given the promise and challenge of site-selective catalysis with peptide or protein recognition domains, ${ }^{7}$ we have initiated a research program to develop a class of catalysts that instead rely upon nucleic acid binding domains. Specifically, we aim to develop enzyme mimics by covalently linking selective, high-affinity DNA aptamers to versatile, efficient small molecule catalysts (Fig. 1b). The resulting DNA-conjugated small molecule
Smith College, Department of Chemistry, Northampton, MA 01063, USA. E-mail: dgorin@smith.edu

$\dagger$ Electronic supplementary information (ESI) available. See DOI: $10.1039 / \mathrm{c} 7 \mathrm{sc} 04554 \mathrm{a}$ 
a)

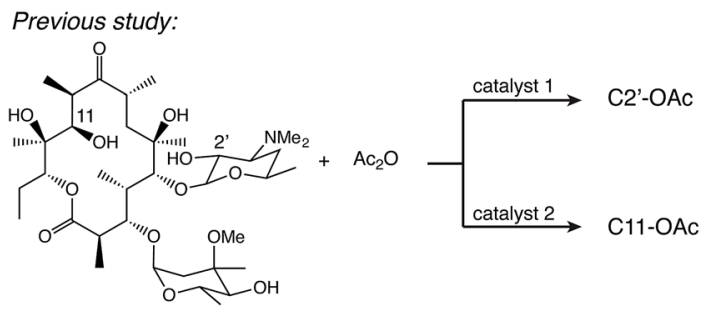

b)

This report:
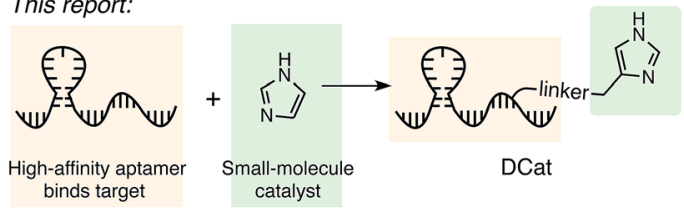

c)

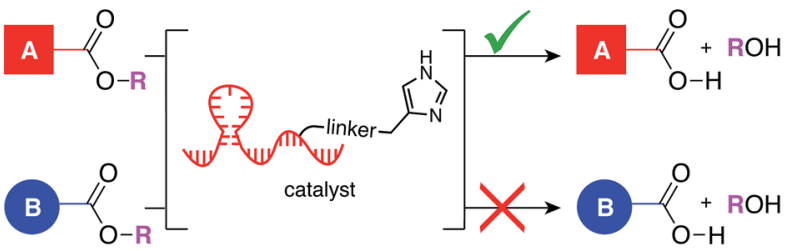

Fig. 1 (a) Miller's catalyst-controlled, site-selective acetylation of erythromycin. ${ }^{1}$ (b) DCats assembled from small molecule catalyst (imidazole) and DNA aptamer. (c) DCat-catalyzed, site-selective ester hydrolysis.

catalysts (DCats) hold potential as a general class of siteselective reagents (Fig. 1c) for a wide range of reactions.

Nucleic acids have found some use as tools to control reaction selectivity in organic synthesis; well-developed examples include DNA-templated synthesis to control reactions of DNA-linked substrates ${ }^{10}$ and the use of DNA as a chiral ligand for enantioselective catalysis. ${ }^{11}$ The use of an RNA aptamer as a stoichiometric, non-covalent protecting group seminally demonstrated the potential of nucleic acids to change reaction site-selectivity within a non-DNA-linked substrate. ${ }^{12}$ Site-selective acylation of aminoglycoside antibiotics was achieved through blocking of some amines by the bound aptamer, which increased the relative reactivity of the remaining solvent-accessible amines.

Although limitedly studied, hybrid catalysts assembled from a nucleic acid recognition domain and a second reactive domain have demonstrated promising reactivity. ${ }^{13}$ In 2008, Marx and Hartig reported that proline linked to porphyrin-binding ssDNA effectively catalyzes the aldol reaction of a porphyrin-aldehyde. ${ }^{13 a}$ Although significant rate enhancement for the reaction of the porphyrin-aldehyde was observed with DNA-linked proline relative to free-proline, no studies with ostensibly non-binding aldehyde substrates were done. Very recently, Willner demonstrated that an aptamer-linked DNAzyme showed enhanced catalysis compared with the free DNAzyme. ${ }^{13 b, 14}$ Furthermore, the rate of reaction correlated with the binding affinity of the aptamer for its target, strongly suggesting that aptamers could be used as recognition domains in site-selective reagents and that selecting and/or designing binding and catalytic function separately can result in effective rate enhancement. ${ }^{15}$

Herein, we report a DNA-imidazole conjugate (DCat1) able to site-selectively increase the rate of ester hydrolysis in a cholic acid-derived ester (1); other esters not incorporating a cholate moiety are unaffected by the DCat. ${ }^{16}$ Ester hydrolysis was chosen for initial study due to its broad potential applicability, such as in the activation of caged probe molecules or pro-drugs, ${ }^{17}$ or in the functional perturbation of bioactive esters, such as $\mathrm{N}$-acyl-homoserine lactone signals in quorum sensing. ${ }^{18}$ The DCat-catalyzed hydrolysis is dependent upon aptamer folding, a phenomenon which we exploited in the design and validation of a stimulus-responsive DCat that can be "turned on" by a preprogrammed signaling molecule. Analysis of hydrolysis kinetics reveals that DCat1 is 100 times more effective per mole than free small molecule catalyst, and comparison of target and nontarget esters illustrates that the DCat is highly site-selective.

\section{Results and discussion}

\section{Design and synthesis of DCats for ester hydrolysis}

In principle, either DNA or RNA aptamers may be incorporated into DCats. DNA has several technical advantages over RNA, including greater stability, and is therefore a more promising scaffold for interfacing with other reaction chemistries, even including transition metal catalysis. ${ }^{\mathbf{1 0 , 1 4 b}}$ Cholic acid-derived umbelliferone ester $\mathbf{1}$ was chosen as the target for these proof of principle studies (Fig. 2a) since several aptamers that selectively bind cholic acid have been reported $^{20}$ and the fluorogenic umbelliferone ester enables convenient determination of reaction progress. ${ }^{21}$ Imidazole was chosen as the hydrolytic small molecule catalyst due to its demonstrated compatibility with DNA. ${ }^{\mathbf{1 6}}$

DCats were modularly assembled by conjugation of histamine to high-affinity, amine-modified cholic acid aptamers via the DSG crosslinker (see ESI, Fig. S1 $\dagger$ ). Two DNA sequences were used (Ap1, 48-mer, $K_{\mathrm{D}}=5 \mu \mathrm{M}$ and Ap2, 40-mer, $K_{\mathrm{D}}=27.8 \mu \mathrm{M}$ ); both are proposed to bind cholic acid at a three-way junction site (as depicted in Fig. 2b). ${ }^{20}$ Since the optimal attachment point for the imidazole catalyst was uncertain, a small library of DCats was constructed from aptamers with different amine modification sites distributed around the proposed binding site (Fig. 2b).

\section{DCats show enhanced catalytic activity}

To investigate the catalytic activity of each DCat, the initial rate of the DCat-catalyzed hydrolysis of 1 ([DCat] $=5 \mu \mathrm{M},[1]=$ $10 \mu \mathrm{M}$ ) was compared with the initial rate of the same reaction catalyzed by free imidazole ([imidazole] $=5 \mu \mathrm{M})$. Since each DCat molecule contains one imidazole moiety, this experiment illustrates whether the DNA influences the rate of imidazolecatalyzed hydrolysis; if no rate enhancement is conferred by the DNA domain, then the reaction rate should match that observed with free imidazole. For additional comparisons, hydrolysis of 1 with no catalyst and increasing concentrations of free imidazole $(100 \mu \mathrm{M}, 500 \mu \mathrm{M}$ and $1 \mathrm{mM})$ was also performed (Fig. S4 $\dagger$ ). Fluorescence from liberated umbelliferone, a reaction product, was monitored over time (Fig. 2c).

Strikingly, several DCats in the library, including the five shown in Fig. 2c, showed significant rate enhancement compared with equimolar imidazole. The reactions with 
imidazole at varying concentrations provide a further benchmark to evaluate the relative rates of hydrolysis. Several DCats (DCat2, DCat4 and DCat7) at $5 \mu \mathrm{M}$ concentration catalyze ester hydrolysis at initial rates comparable with $100 \mu \mathrm{M}$ (20-fold excess) free imidazole, while the rate with DCat10 is even faster. However, the most effective construct, DCat1, hydrolyzes 1 at an initial rate comparable to $500 \mu \mathrm{M}$ (100-fold excess) untethered imidazole (Fig. 2c and d).

To confirm this qualitative assessment, the effective first order rate constants were determined for each reaction by dividing the slope of the linear region at early times by the concentration of substrate $\left(k_{\text {obs }}\right.$, Table 1$)$. The pseudo firstorder rate constants for $5 \mu \mathrm{M}$ DCat1 and equimolar imidazole differ by an order of magnitude $\left(k_{\mathrm{obs}}=0.09 \mathrm{~h}^{-1} v s\right.$. $0.009 \mathrm{~h}^{-1}$ ). However, $5 \mu \mathrm{M}$ of imidazole does not accelerate hydrolysis significantly above the background (uncatalyzed) rate, so a more informative comparison is $5 \mu \mathrm{M}$ DCat1 $v s$. an equally effective concentration of imidazole. Notably, the rate constant for $500 \mu \mathrm{M}$ imidazole is identical to that for $5 \mu \mathrm{M}$ DCat1 $\left(0.11 \mathrm{~h}^{-1}\right.$ vs. $\left.0.09 \mathrm{~h}^{-1}\right)$. That is, DCat1 is as effective as a 100-fold excess of free small molecule catalyst. This becomes more apparent when the second-order rate constants are extrapolated from the effective first order rates. ${ }^{22}$ Both 5 and $500 \mu \mathrm{M}$ imidazole yield second order rate constants $\sim 0.2 \mathrm{mM}^{-1} \mathrm{~h}^{-1}$, while $k_{\text {app }}$ for DCat1 is two orders of magnitude larger $\left(k_{\text {app }}=17 \mathrm{mM}^{-1} \mathrm{~h}^{-1}\right) .{ }^{23}$ Taken together, these experiments show that linking the DNA aptamer to imidazole dramatically increases the rate of ester hydrolysis of 1 compared with free imidazole, and that this rate enhancement varies with the site of DNA modification.

\section{DCat catalysis is dependent on DNA tertiary structure}

We hypothesized that the enhanced rate of ester hydrolysis observed upon tethering imidazole to the DNA aptamer is due to binding of the aptamer to the cholate moiety in $\mathbf{1}$, which increases the effective concentration of imidazole and $\mathbf{1 .}^{\mathbf{1 0}}$ Because aptamer function is known to depend on the ssDNA folding into the active three-dimensional structure, this suggests that DCat activity requires aptamer folding. To test this, DCat-catalyzed hydrolysis of $\mathbf{1}$ was conducted in the presence of an ssDNA complementary to the aptamer (comp1, Fig. 3). ${ }^{24}$ The addition of equimolar complementary DNA $([$ comp1 $]=5 \mu \mathrm{M})$ to the standard reaction conditions $([\mathrm{DCat}]=$ $5 \mu \mathrm{M},[1]=10 \mu \mathrm{M})$ resulted in complete loss of DCat1 activitythe hydrolysis of $\mathbf{1}$ proceeded identically to the reaction with $5 \mu \mathrm{M}$ free imidazole (which is also indistinguishable from the reaction run without any added catalyst). A control experiment demonstrated that addition of a non-complementary ssDNA (random DNA) had no effect on the rate of hydrolysis; the DCatmediated reaction proceeded rapidly just as in the case where no additional DNA was added. ${ }^{25}$ These results indicate that correctly-folded aptamer is necessary for catalysis and strongly suggests that the aptamer domain of DCat1 binds to 1 during the catalytic cycle.

The "turn-off" response of DCat1 to the presence of comp1 suggests that DCats have the potential to be stimuli-responsive,

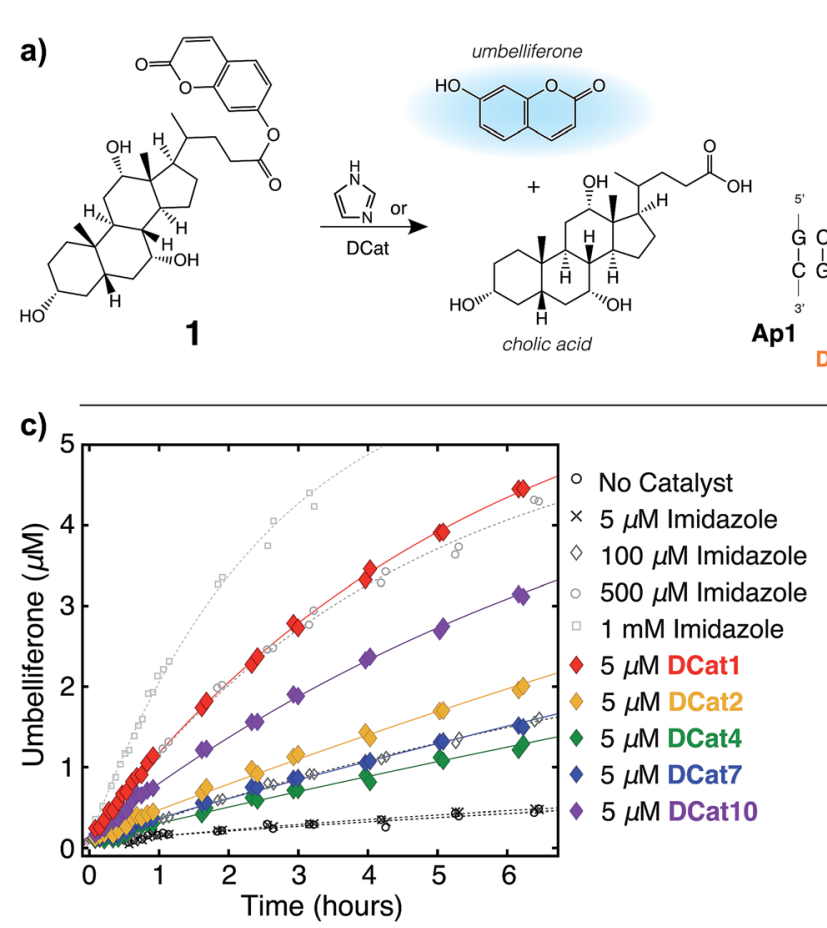

b)
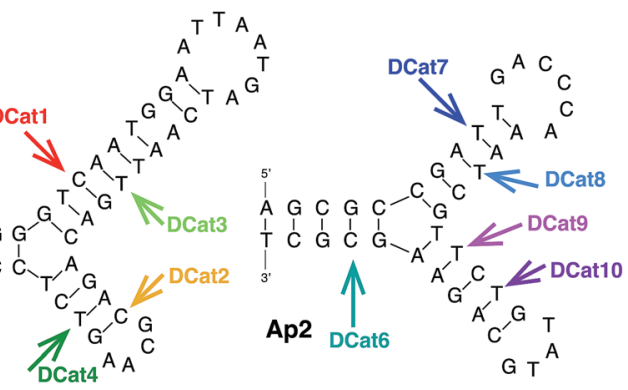

d)

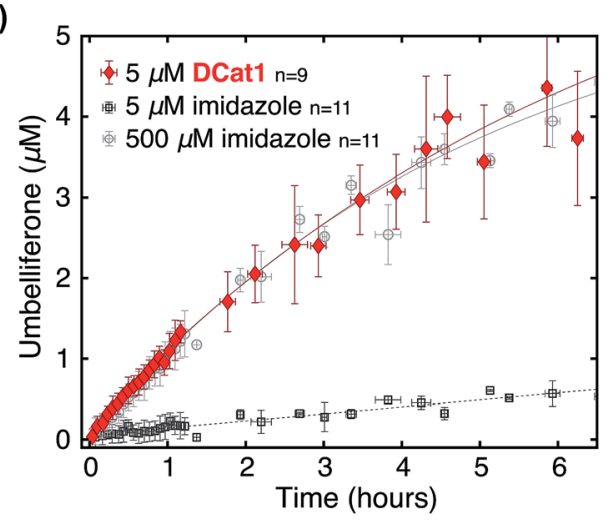

Fig. 2 DCat enhances catalytic activity: (a) fluorogenic hydrolysis of 1 catalyzed by imidazole or a DCat. (b) Small DCat library constructed from two DNA sequences (Ap1 and Ap2) with various imidazole attachment sites. Predicted secondary structures were found using Mfold. ${ }^{19}$ (c) Product formation over time from hydrolysis of 1 by each DCat (colored diamond markers). Control experiments with specified concentrations of free, untethered imidazole as shown (grey marker symbols). (d) Replicates and error bars for hydrolysis with DCat1 and benchmark imidazole concentrations. Error bars indicate a standard deviation. See ESI $\uparrow$ for further details. 
Table 1 Effective first-order kinetic constants for DCat1 and free imidazole. DCat1 $(n=9)$, imidazole $(n=11)$. Error is defined as one standard deviation

\begin{tabular}{lcc}
\hline Catalyst & $k_{\text {obs }}{ }^{a}\left(\mathrm{~h}^{-1}\right)$ & $k_{\text {app }}{ }^{b}\left(\mathrm{mM}^{-1} \mathrm{~h}^{-1}\right)$ \\
\hline $5 \mu$ M DCat1 & $0.09 \pm 0.03$ & $17 \pm 5$ \\
$500 \mu$ M imidazole & $0.11 \pm 0.04$ & $0.21 \pm 0.07$ \\
$5 \mu$ M imidazole & $0.009 \pm 0.003$ & $0.18 \pm 0.07$
\end{tabular}

${ }^{a}$ Observed pseudo first-order rate constant determined from the initial reaction rate: $k_{\mathrm{obs}}=v_{\mathrm{i}} /\left[S_{0}\right]=v_{\mathrm{i}} / 10 \mu \mathrm{M}{ }^{b}{ }^{b}$ Apparent, extrapolated secondorder rate constant determined by: $k_{\mathrm{app}}=\left(k_{\mathrm{obs}}-k_{\mathrm{back}}\right) /[$ catalyst $]$ where $k_{\text {back }}=0.008 \pm 0.002 \mathrm{~h}^{-1}$, the uncatalyzed hydrolysis rate.

dynamic reagents that can be controllably and reversibly deactivated and activated. To demonstrate this, a system for controllable, time-dependent DCat activation was developed using toehold displacement (Fig. 3a). ${ }^{26}$ In this assay, the DCat is initially incubated with comp1 before the addition of substrate (Fig. 3a). Hybridization inhibits DCat activity, as described above. The hybridized complementary strand contains an extra toehold region of 10 nucleotides which provides an opportunity for strand displacement. When rescue1, ssDNA with a primary sequence that is fully complementary to comp1, is added, it will displace DCat1, which should rescue DCat function and "turn on" the hydrolysis of $\mathbf{1}$. Delightfully, after incubation of DCat with comp1, DCatcatalyzed hydrolysis at enhanced rates was observed only upon addition of the rescue1 strand (Fig. 3b). This was demonstrated at two different time points ( 0.55 hours and 2.1 hours) to illustrate temporal stimulus-responsive control of DCat activity. This type of behavior is highly desirable for a variety of applications in DNA nanotechnology. ${ }^{27}$ A DCat could be easily incorporated into other nucleic acid basedarchitectures, including hydrogels, DNA origami, and other nanostructures, expanding the possible applications of DCats, and offering an avenue for incorporating small molecule catalysts into DNA architecture and nanotechnology.

\section{DCat shows site-selective rate enhancement}

Next, the site-selectivity of DCat1 was investigated by comparing the rate of DCat-mediated hydrolysis of $\mathbf{1}$ with the rates of other umbelliferone esters. The results presented thus far suggest that target recognition and binding is essential for the enhanced rate of DCat-catalyzed hydrolysis of $\mathbf{1}$, and therefore other esters not recognized by the DCat's DNA aptamer domain should not be affected. To test this hypothesis, the DCat1catalyzed hydrolysis of esters $\mathbf{2}$ and 3, derived from acetic acid and adenosine, respectively, were investigated (Fig. 4a and b). For all three esters $(\mathbf{1}, \mathbf{2}, \mathbf{3})$, uncatalyzed background hydrolysis is indistinguishable from reactions containing $5 \mu \mathrm{M}$ imidazole (see Table 2 and ESI $\dagger$ ) so enhancement above $5 \mu \mathrm{M}$ imidazole signifies meaningful rate increase. Notably, no rate enhancement from the DCat is observed in reactions with 2 or 3 (Table 2); the rates of hydrolysis are very similar whether the esters are treated with $5 \mu \mathrm{M}$ DCat1 or $5 \mu \mathrm{M}$ free imidazole (Fig. $4 \mathrm{c}$ and d). In contrast, the rate of hydrolysis of $\mathbf{1}$ is clearly a)
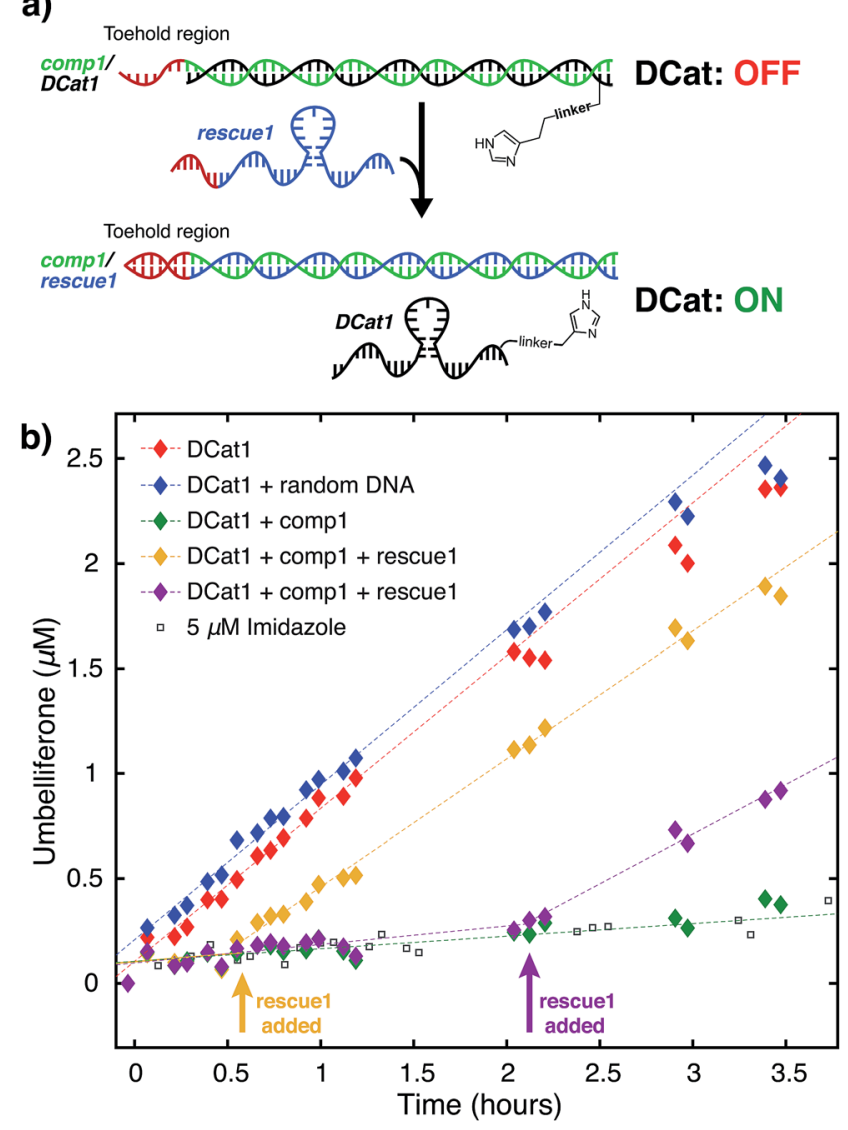

Fig. 3 DCat "turn-on" using toehold displacement: (a) inactive DCat1 rescued by displacement of comp1 with rescue1. (b) Product formation over time from hydrolysis of 1 by DCat1 in the presence (green diamond) and absence of comp1. DCat activity "turns on" at 35 minutes (yellow diamonds) or 130 minutes (purple diamonds) upon addition of rescue1.

enhanced in the presence of DCat1, which illustrates siteselectivity for $\mathbf{1}$, and not 2 or 3 .

To quantify these observations, a rate enhancement was determined for each catalyst-ester pair by taking the observed first-order rate constant and dividing by the background reaction, $\left(k_{\mathrm{obs}} / k_{\mathrm{back}}\right)$. At the lowest concentration of $5 \mu \mathrm{M}$, imidazole has $k_{\text {obs }} / k_{\text {back }}$ of $\sim 1$ regardless of the ester, corresponding to a lack of rate enhancement. Imidazole at higher concentration gives rate enhancement across all three substrates; no appreciable site selectivity is observed. In contrast, the rate enhancements for DCat1 are negligible for hydrolysis of the non-target esters 2 and $3\left(k_{\text {obs }} / k_{\text {back }}=1.2\right.$ and 0.9 respectively), but significant for hydrolysis of $1\left(k_{\mathrm{obs}} / k_{\mathrm{back}}=12\right)$. This demonstrates a high degree of site-selectivity, as all three molecules contain umbelliferone esters, but only one undergoes rapid hydrolysis. Excitingly, this highlights the potential use of DCats as site-selective reagents in mixtures.

\section{Kinetic analysis}

The results above are broadly consistent with non-covalent binding of DCat to the substrate as a key step in the catalytic 
cycle. This suggests an analogy to protein enzymes, where formation of an enzyme-substrate complex prior to covalent transformation is generally proposed. ${ }^{28}$ If the reactions proceed within an overall mechanistic framework closely resembling natural protein enzymes, DCats may be considered as modularly assembled synthetic enzymes, and the reaction kinetics with DCats should closely resemble enzyme kinetics (Fig. 5a). To test this, the DCat1-catalyzed ester hydrolysis was investigated over a range of substrate concentrations $([1]=10-75 \mu \mathrm{M}$ etc. $)$, initial rate constants were determined (Fig. S5 $\dagger$ ), and the results were fit to the Michaelis-Menten equation (Fig. 5). From this, $k_{\text {cat }}\left(0.8 \mathrm{~h}^{-1}\right)$ and $K_{\mathrm{M}}(26 \mu \mathrm{M})$ were determined. The $K_{\mathrm{M}}$ value is consistent with the reported $K_{\mathrm{D}}$ of the cholic acid aptamer. $^{20}$ The $k_{\text {cat }}$ value is small compared with natural enzymes, ${ }^{29}$ but higher than many DNAzymes ${ }^{14 b}$ and de novo designed enzymes. ${ }^{\mathbf{1 3 b}}$ Furthermore, kinetic comparison of DCat1 with free imidazole highlights significant rate advantages for the DCat.

To better compare the mechanisms of the DCat-catalyzed hydrolysis and the reaction catalyzed by free imidazole, the imidazole-catalyzed reaction $([$ imidazole $]=500 \mu \mathrm{M})$ was also investigated at multiple substrate concentrations (Fig. 5). The imidazole-catalyzed reaction exhibited second-order kinetics (see ESI $\dagger$ ), so each single run fits a simple pseudo first-order kinetic model. ${ }^{14 b, d}$ As expected for a typical second-order kinetic reaction, the initial rate increases linearly as [1] increases. The pseudo first-order rate constant $\left(k_{\text {obs }}\right)$ is $0.15 \mathrm{~h}^{-1}$ and no saturation kinetics are observed. Comparison of the reaction rates at high substrate concentrations clearly illustrates that the DCat kinetic model (which shows saturation) is completely different
Table 2 Rate Enhancement calculated for each ester with DCat1 and imidazole. Error is defined as one standard deviation

\begin{tabular}{llcc}
\hline & \multicolumn{2}{c}{ Rate enhancement: $k_{\text {obs }} / k_{\text {back }}{ }^{a}$} & \\
\cline { 2 - 4 } Catalyst & Substrate 1 & Substrate 2 & Substrate 3 \\
\hline $5 \mu$ M DCat1 & $12 \pm 4$ & $1.2 \pm 0.5$ & $0.9 \pm 0.5$ \\
$500 \mu$ M imidazole & $15 \pm 5$ & $16 \pm 3$ & $8 \pm 3$ \\
$5 \mu$ M imidazole & $1.1 \pm 0.3$ & $1.1 \pm 0.5$ & $1.1 \pm 0.5$
\end{tabular}

${ }^{a}$ Rate enhancement is the ratio of the pseudo first-order rate constant of a catalyzed reaction, $k_{\text {obs }}$, divided by $k_{\text {back }}$ where $k_{\text {back }}$ is the selfhydrolysis rate in the presence of no catalyst. For $10 \mu \mathrm{M}$ 1: $k_{\text {back }}=$ $0.008 \pm 0.002 \mathrm{~h}^{-1}$; for $2: k_{\text {back }}=0.013 \pm 0.008 \mathrm{~h}^{-1}$; for $3: k_{\text {back }}=$ $0.011 \pm 0.008 \mathrm{~h}^{-1}$.

from free imidazole, despite the fact that an imidazole moiety is crucial to reaction progress in both cases.

At low substrate concentration, the "first-order region" of Michaelis-Menten kinetics, both the DCat and imidazole catalyzed reactions may be considered pseudo first-order reactions and thereby meaningfully compared..$^{30}$ The $500 \mu \mathrm{M}$ imidazolecatalyzed reaction follows a pseudo first-order $k_{\mathrm{obs}}^{\prime}=0.15 \mathrm{~h}^{-1}$, while catalysis with $5 \mu \mathrm{M}$ DCat1 gives $k_{\text {obs }}^{\prime}=k_{\text {cat }}\left[E_{0}\right] / K_{\mathrm{M}}=$ $0.15 \mathrm{~h}^{-1}$. The identical $k_{\text {obs }}$ obtained for each reaction are consistent with the observations in Fig. 2; the rate of product formation is the same. Additionally, the effective first-order rate constant for DCat1 from Table 1 also agrees with the first-order region of this Michaelis-Menten description. When the second order rate constants are extrapolated for each catalyst, the rate constant for imidazole is found to be $k_{\text {app }}^{\prime} \approx$ $0.3 \mathrm{mM}^{-1} \mathrm{~h}^{-1}$ while DCat1 gives $k_{\mathrm{app}}^{\prime} \approx 30 \mathrm{mM}^{-1} \mathrm{~h}^{-1}$ a)

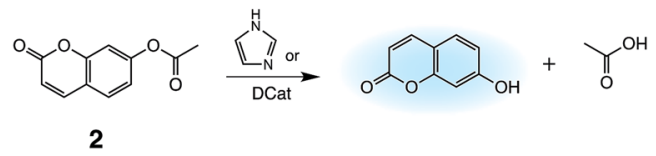

c)

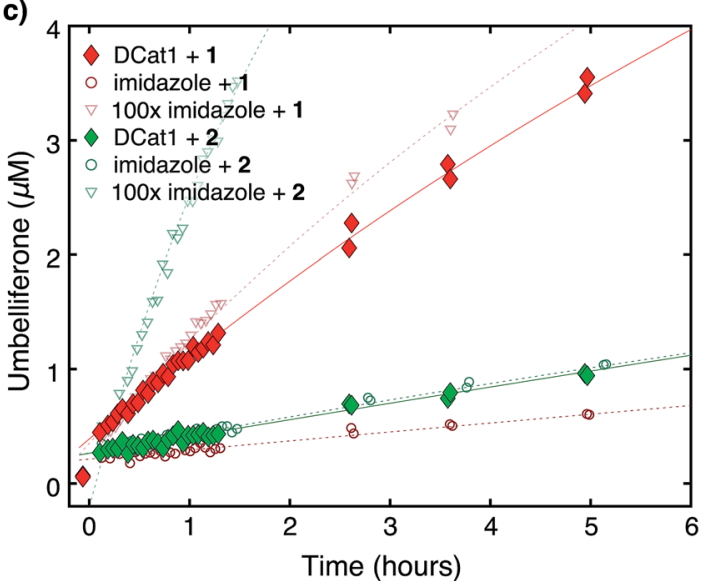

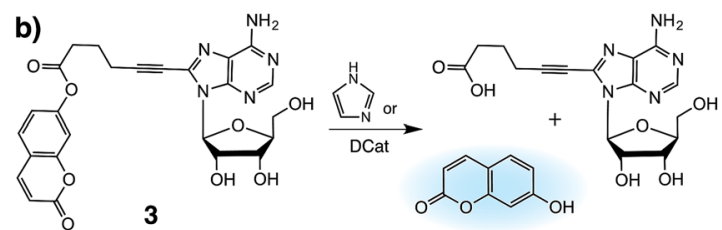

d)

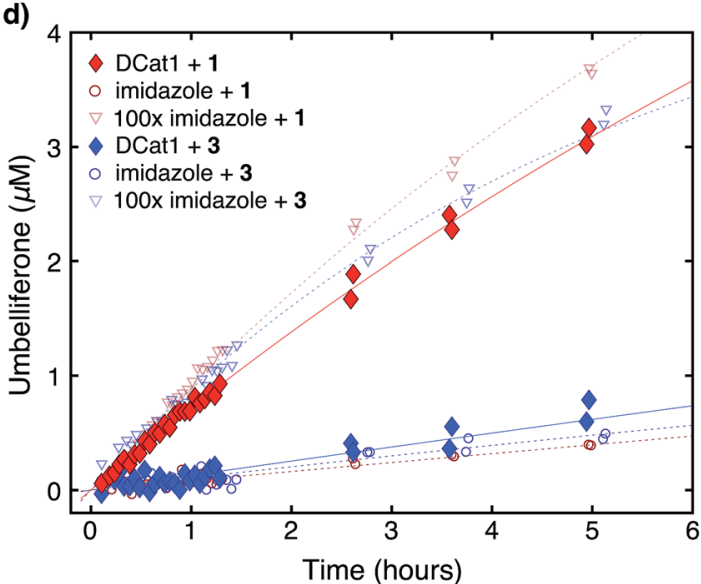

Fig. 4 Site-selective ester hydrolysis (a) fluorogenic hydrolysis of 2. (b) Fluorogenic hydrolysis of 3. (c) DCat1-catalyzed reaction of 1 (red-filled diamonds) vs. 2 (green-filled diamonds). Reactions with benchmark imidazole concentrations also shown (open shapes). (d) DCat1-catalyzed reaction of 1 (red-filled diamonds) vs. 3 (blue-filled diamonds). Reactions with benchmark imidazole concentrations also shown (open shapes). See ESI† for further details. 
a)

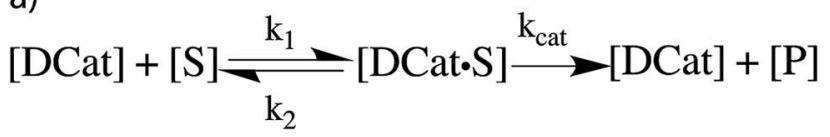

b)

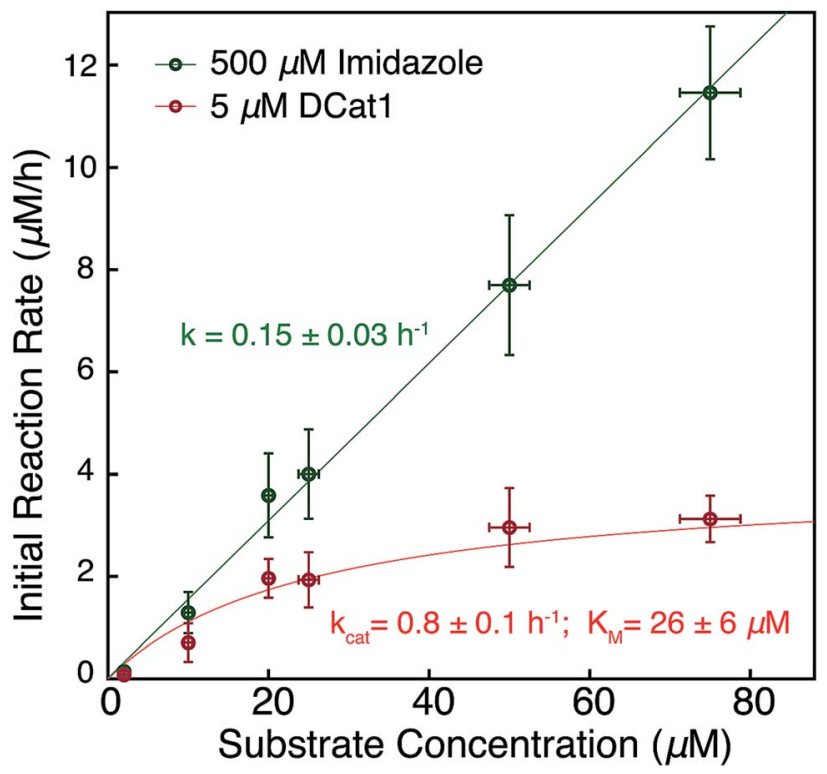

Fig. 5 Proposed enzyme-like mechanism for DCat catalysis (a) Michaelis-Menten model (b) dependence of initial reaction rate on substrate concentration for DCat-catalysis (red) with MichaelisMenten parameters vs. imidazole (green) catalysis with pseudo firstorder rate constant. Error bars \pm 1 S.D. See ESI† for further details.

(Table 3). That is, the intrinsic rate for DCat1-catalyzed hydrolysis of $\mathbf{1}$ is two orders of magnitude higher than that of free imidazole. These results are very encouraging for the potential application of DCats to biolabeling. In biological mixtures, the desired target molecule is often low $(0.1-50 \mu \mathrm{M}$ range for proteins in the cytoplasm $)^{31}$ so the rate enhancement conferred by a DCat at low substrate concentrations (in the Michaelis-Menten "first order region") is highly relevant and promising.

\section{Conclusions}

Synthetic catalysts that incorporate molecular recognition domains offer a strategy for achieving the site-selective transformation of one instance of a functional group. In this work, we demonstrated that a DCat, prepared by tethering imidazole to a DNA aptamer, increases the rate of ester hydrolysis of a specific target ester by $>100$-fold compared with equimolar untethered imidazole. No rate enhancement was observed with other esters, demonstrating site-selective catalysis. DCatcatalyzed hydrolysis followed enzyme-like kinetics at biologically relevant concentrations, and a stimuli-responsive, programmable variant of the DCat system exploiting toehold displacement was demonstrated. The fact that a DCat can be temporally controlled in this manner indicates that the system may be useful in drug-release and related applications.
Table 3 Michaelis-Menten Analysis of DCat1 Compared with imidazole. The Michaelis-Menten parameters are used to compare $5 \mu \mathrm{M}$ DCat1 $(n=4)$ to $500 \mu \mathrm{M}$ imidazole $(n=6)$ kinetic behavior over increasing substrate concentration. Error is defined by one standard deviation

\begin{tabular}{lllll}
\hline Catalyst & $k_{\text {cat }}\left(\mathrm{h}^{-1}\right)$ & $K_{\mathrm{M}}(\mu \mathrm{M})$ & $\begin{array}{c}k_{\text {app }}{ }^{a} \\
\left(\mathrm{mM}^{-1} \mathrm{~h}^{-1}\right)\end{array}$ & $k_{\text {app }}^{\prime} / k_{\text {app,imid }}^{\prime}$ \\
\hline $\begin{array}{l}500 \mu \mathrm{M} \text { imidazole } \\
5 \mu \mathrm{M} \text { DCat1 }\end{array}$ & - & - & $0.30 \pm 0.07$ & 1 \\
$0.8 \pm 0.1$ & $26 \pm 6$ & $31 \pm 8$ & 103 \\
${ }^{a}$ Extrapolated apparent second-order rate constant determined by $k_{\text {app }}^{\prime}$ \\
$=k_{\text {obs } /[\text { catalyst] }}$
\end{tabular}

In analogy to natural protein enzymes that both recognize their targets and catalyze specific chemical reactions, we have assembled an enzyme mimic that uses a DNA aptamer as a recognition domain while exploiting the efficiency and versatility of a small molecule catalyst. The use of functional nucleic acids as the binding domain promises that DCats can be prepared for a wide range of targets and applications, since SELEX enables the discovery of aptamers that bind nearly any target. Furthermore, both the aptamer and catalyst can be modularly substituted as necessary for a particular application or reaction chemistry. We envision that DCats may be useful in a broad array of applications, including in target-oriented synthesis to differentiate two instances of the same functional group within a single complex molecule and in bioconjugation efforts to target a particular protein or metabolite in a complex biological mixture. Experimental efforts to expand the scope of reactions mediated by DCats and to demonstrate selectivity in complex biological mixtures and other applications are currently underway.

\section{Conflicts of interest}

There are no conflicts of interest to declare.

\section{Acknowledgements}

We gratefully acknowledge financial support from the National Science Foundation (CAREER 1554814), TriLink BioTechnologies, the Research Corporation Cottrell College Science Award, and Smith College for a Jean Picker Fellowship (D. J. G.). We also thank Smith College for Summer fellowships (A. E. A., D. E. C., J. K., J. N. K., S. L., Y. Y., and Y. Z.), a McKinley Honors Fellowship (A. E. A.), and Tomlinson funds (A. E. A., D. E. C., J. K., and Y. Z.) to support undergraduate researchers. Dr Charles Amass, Lou Ann Bierwert, and Dr Kalina Dimova (Smith College) assisted with instrumentation. We also thank Professor David Bickar (Smith College) for discussions on the kinetic analysis. High-resolution mass spectral data were obtained at the University of Massachusetts Mass Spectrometry Center.

\section{References}

1 C. A. Lewis and S. J. Miller, Angew. Chem., 2006, 118, 57445747. 
2 H. M. L. Davies and D. Morton, J. Org. Chem., 2016, 81, 343350.

3 (a) C. R. Bertozzi and P. Wu, Curr. Opin. Chem. Biol., 2013, 17, 717-718; (b) E. M. Sletten and C. R. Bertozzi, Angew. Chem., Int. Ed., 2009, 48, 6974-6998; (c) K. L. Kiick, E. Saxon, D. A. Tirrell and C. R. Bertozzi, Proc. Natl. Acad. Sci. U. S. A., 2002, 99, 19-24; (d) B. A. Griffin, S. R. Adams and R. Y. Tsien, Science, 1998, 281, 269-272; (e) S. Lin, X. Yang, S. Jia, A. M. Weeks, M. Hornsby, P. S. Lee, R. V. Nichiporuk, A. T. Iavarone, J. A. Wells, F. D. Toste and C. J. Chang, Science, 2017, 355, 597-602.

4 (a) M. S. Chen and M. C. White, Science, 2010, 327, 566-571; (b) M. S. Chen and M. C. White, Science, 2007, 318, 783-787; (c) J. I. MacDonald, H. K. Munch, T. Moore and M. B. Francis, Nat. Chem. Biol., 2015, 11, 326-331. For reviews, see: (d) C. R. Shugrue and S. J. Miller, Chem. Rev., 2017, 117, 11894-11951; (e) F. D. Toste, M. S. Sigman and S. J. Miller, Acc. Chem. Res., 2017, 50, 609-615.

5 J. J. Perona and C. S. Craik, Protein Sci., 1995, 4, 337-360.

6 M. McClelland, M. Nelson and E. Raschke, Nucleic Acids Res., 1994, 22, 3640-3659.

7 (a) T. P. Pathak and S. J. Miller, J. Am. Chem. Soc., 2012, 134, 6120-6123; (b) F. Vohidov, J. M. Coughlin and Z. T. Ball, Angew. Chem., Int. Ed., 2015, 54, 4587-4591; (c) Z. Chen, B. V. Popp, C. L. Bovet and Z. T. Ball, ACS Chem. Biol., 2011, 6, 920-925; (d) J. Ohata and Z. T. Ball, J. Am. Chem. Soc., 2017, 139, 12617-12622; (e) T. Suzuki, Y. Ota, Y. Kasuya, M. Mutsuga, Y. Kawamura, H. Tsumoto, H. Nakagawa, M. G. Finn and N. Miyata, Angew. Chem., Int. Ed., 2010, 49, 6817-6820; (f) S. Han and S. J. Miller, J. Am. Chem. Soc., 2013, 135, 12414-12421; $(g)$ J. Gatto Gregory, M. T. Boyne, N. L. Kelleher and C. T. Walsh, J. Am. Chem. Soc., 2006, 128, 3838-3847.

8 (a) S. Tsukiji, M. Miyagawa, Y. Takaoka, T. Tamura and I. Hamachi, Nat. Chem. Biol., 2009, 5, 341-343; (b) T. Hayashi, Y. Sun, T. Tamura, K. Kuwata, Z. Song, Y. Takaoka and I. Hamachi, J. Am. Chem. Soc., 2013, 135, 12252-12258; (c) T. Hayashi, Y. Yasueda, T. Tamura, Y. Takaoka and I. Hamachi, J. Am. Chem. Soc., 2015, 137, 5372-5380.

9 (a) T. K. Sharma, J. G. Bruno and A. Dhiman, Biotechnol. Adv., 2017, 35, 275-301; (b) D. S. Wilson and J. W. Szostak, Annu. Rev. Biochem., 1999, 68, 611-647.

10 (a) X. Li and D. R. Liu, Angew. Chem., Int. Ed., 2004, 43, 48484870; (b) Z. J. Gartner, B. N. Tse, R. Grubina, J. B. Doyon, T. M. Snyder and D. R. Liu, Science, 2004, 305, 1601-1605; (c) C. B. Rosen, T. Tørring and K. V. Gothelf, in Nucleic Acid Nanotechnology, Springer, Berlin, Heidelberg, 2014, pp. 173-197.

11 (a) N. Duchemin, I. Heath-Apostolopoulos, M. Smietana and S. Arseniyadis, Org. Biomol. Chem., 2017, 15, 7072-7087; (b) A. J. Boersma, R. P. Megens, B. L. Feringa and G. Roelfes, Chem. Soc. Rev., 2010, 39, 2083-2092.

12 A. A. Bastian, A. Marcozzi and A. Herrmann, Nat. Chem., 2012, 4, 789-793.

13 Hybrid catalysts with nucleic acid recognition domains: $(a)$ Z. Tang, D. P. N. Gonçalves, M. Wieland, A. Marx and
J. S. Hartig, ChemBioChem, 2008, 9, 1061-1064; (b) E. Golub, H. B. Albada, W.-C. Liao, Y. Biniuri and I. Willner, J. Am. Chem. Soc., 2016, 138, 164-172; (c) G. J. Tong, S. C. Hsiao, Z. M. Carrico and M. B. Francis, J. Am. Chem. Soc., 2009, 131, 11174-11178.

14 (Deoxy)ribozymes: (a) S. K. Silverman, Trends Biochem. Sci., 2016, 41, 595-609; (b) M. Chandra and S. K. Silverman, J. Am. Chem. Soc., 2008, 130, 2936-2937; (c) K. Schlosser, S. A. McManus, and Y. Li, in The Aptamer Handbook: Functional Oligonucleotides and Their Applications, ed. S. Klussmann, Wiley-VCH Verlag GmbH \& Co. KGaA, Weinheim, 2006, pp. 228-261; (d) B. Seelig and A. Jäschke, Chem. Biol., 1999, 6, 167-176.

15 During the preparation of this manuscript, a related application of aptamers for bioconjugation was reported: C. Cui, H. Zhang, R. Wang, S. Cansiz, X. Pan, S. Wan, W. Hou, L. Li, M. Chen, Y. Liu, X. Chen, Q. Liu and W. Tan, Angew. Chem., Int. Ed., 2017, 56, 11954-11957.

16 (a) Z. Ma and J.-S. Taylor, Proc. Natl. Acad. Sci. U. S. A., 2000, 97, 11159-11163; (b) S. W. Santoro, G. F. Joyce, K. Sakthivel, S. Gramatikova and C. F. Barbas, J. Am. Chem. Soc., 2000, 122, 2433-2439; (c) L. Lermer, Y. Roupioz, R. Ting and D. M. Perrin, J. Am. Chem. Soc., 2002, 124, 9960-9961.

17 (a) J. Li and P. R. Chen, Nat. Chem. Biol., 2016, 12, 129-137; (b) S. S. Chandran, K. A. Dickson and R. T. Raines, J. Am. Chem. Soc., 2005, 127, 1652-1653; (c) L. D. Lavis, ACS Chem. Biol., 2008, 3, 203-206.

18 M. E. A. Churchill and L. Chen, Chem. Rev., 2011, 111, 68-85. 19 M. Zuker, Nucleic Acids Res., 2003, 31, 3406-3415.

20 (a) T. Kato, T. Takemura, K. Yano, K. Ikebukuro and I. Karube, Biochim. Biophys. Acta Gene Struct. Expr., 2000, 1493, 12-18; (b) T. Kato, K. Yano, K. Ikebukuro and I. Karube, Nucleic Acids Res., 2000, 28, 1963-1968.

21 (a) G. Klein and J.-L. Reymond, Helv. Chim. Acta, 1999, 82, 400-407; (b) J.-P. Goddard and J.-L. Reymond, Trends Biotechnol., 2004, 22, 363-370; (c) D. Wahler and J.-L. Reymond, Curr. Opin. Chem. Biol., 2001, 5, 152-158.

22 Apparent, extrapolated second-order rate constant determined by: $k_{\text {app }}=\left(k_{\text {obs }}-k_{\text {back }}\right) /\left[\right.$ catalyst] where $k_{\text {back }}=$ $0.008 \pm 0.002 \mathrm{~h}^{-1}$, the background, uncatalyzed rate.

23 For validation of catalyst turnover and control experiments demonstrating that the unmodified DNA aptamer shows no rate enhancement, see the ESI. $\dagger$

24 Z. Cao, R. Tong, A. Mishra, W. Xu, G. C. L. Wong, J. Cheng and Y. Lu, Angew. Chem., Int. Ed., 2009, 48, 6494-6498.

25 For results with all library members, see ESI (Fig. S8†).

26 (a) A. A. Green, P. A. Silver, J. J. Collins and P. Yin, Cell, 2014, 159, 925-939; (b) D. Y. Zhang, A. J. Turberfield, B. Yurke and E. Winfree, Science, 2007, 318, 1121-1125; (c) D. Y. Zhang and E. Winfree, J. Am. Chem. Soc., 2009, 131, 17303-17314.

27 (a) D. Y. Zhang and G. Seelig, Nat. Chem., 2011, 3, 103-113; (b) Y. R. Yang, Y. Liu and H. Yan, Bioconjugate Chem., 2015, 26, 1381-1395; (c) H. Bauke Albada, E. Golub and I. Willner, Chem. Sci., 2016, 7, 3092-3101; (d) F. Wang, X. Liu and I. Willner, Angew. Chem., Int. Ed., 2015, 54, 1098-1129; (e) M. Smith, Nature, 2006, 440, 283-284, For 2D and/or 3-D architectures templated by DNA and 
incorporating other biomolecules, see: $(f)$ N. S. Selden, M. E. Todhunter, N. Y. Jee, J. S. Liu, K. E. Broaders and Z. J. Gartner, J. Am. Chem. Soc., 2012, 134, 765-768; $(\mathrm{g})$ K. M. El Muslemany, A. A. Twite, A. M. ElSohly, A. C. Obermeyer, R. A. Mathies and M. B. Francis, J. Am. Chem. Soc., 2014, 136, 12600-12606; (h) R. Wacker and C. M. Niemeyer, ChemBioChem, 2004, 5, 453-459.

28 Engineered protein enzymes that show enzyme-like kinetics: (a) M. E. Wilson and G. M. Whitesides, J. Am. Chem. Soc., 1978, 100, 306-307; (b) P. J. Deuss, R. den Heeten, W. Laan and P. C. Kamer, Chem.-Eur. J., 2011, 17, 4680-4698.
29 A. Bar-Even, E. Noor, Y. Savir, W. Liebermeister, D. Davidi, D. S. Tawfik and R. Milo, Biochemistry, 2011, 50, 4402-4410. 30 This kinetic analysis is distinct and separate from the firstorder $k_{\text {obs }}$ in Table 1. For a discussion on the comparison between first-order and Michaelis-Menten kinetics, see: E. A. Lissi and E. B. Abuin, Langmuir, 2000, 16, 10084-10086. 31 (a) J.-Q. Wu and T. D. Pollard, Science, 2005, 310, 310-314; (b) S. Pedersen, P. L. Bloch, S. Reeh and F. C. Neidhardt, Cell, 1978, 14, 179-190. 